\title{
Acute Respiratory Infections (ARI) and Weaning Status of Infants Admitted in Selected Hospitals of Rajshahi- A Case Control Study
}

\author{
Farhana Yasmin, ${ }^{1}$ Md. Jawadul Haque ${ }^{2}$
}

\begin{abstract}
Background: Acute respiratory infections (ARI) include upper respiratory tract infections and lower respiratory tract infections. Infections of the respiratory tract are perhaps the most common human ailment. While they are a source of discomfort, disability and loss of time for most adults, they are a substantial cause of morbidity and mortality in young children. ARI may cause inflammation of the respiratory tract anywhere from nose to alveoli, with a wide range of combination of symptoms and signs. ARI in children is most common among others because heir constant contact with other kids who could be virus carriers. Children often don't wash their hands regularly. They are also more likely to rub their eyes and put their fingers in their mouths, resulting in the spread of viruses. For prevention of malnutrition and infection of child breast feeding is an important determinant of child health in the prevention of malnutrition and infection but in many cultures other food was introduced years before the cessation of breast feeding. In Bangladesh, many infectious diseases such as diarrhoea and acute respiratory infections are the main cause of mortality and morbidity in infants aged less than one year. The importance of breast feeding in the prevention of infectious diseases during infancy is well known.
\end{abstract}

Objective: In this study our main objective is to evaluate the association between ARI of infant and weaning status of infants admitted in selected Hospitals of Rajshahi. Study place and

Method: This study provided a wide range of information regarding ARI and weaning status of infants admitted in selected hospitals of Rajshahi and from the surroundings. This was a case control type of study. A total of $\mathbf{2 3 0}$ caregiver or mothers were interviewed.

Result \& discussion: It was found that the relationship between weaning status of infants and occurrence of ARI was statistically significant $(p<0.001)$. It was showed that majority of the babies of the case group were weaned by Cow's milk and within the control group by mashed rice. The association between occurrence of ARI and type of weaning food was statistically significant $(p<0.001)$. It was also found that majority of the babies living in rural areas developed ARI and also within the case group majority $(29.6 \%)$ of the babies who were not exclusively breast fed developed ARI and the association between occurrence of ARI and EBF was statistically significant $(p<0.001)$.

Conclusion: Our study suggests that proportion of ARI is more among the infants who were weaned earlier.

Keyword: ARI, weaning food, Adenovirus

TAJ 2019; 32: No-1: 70-81

${ }^{1}$ Lecturer, Department of Community Medicine, Rajshahi Medical College, Rajshahi.

2 Professor, Department of Community Medicine, Rajshahi Medical College, Rajshahi. 


\section{Introduction}

Acute respiratory infection is an infection that may interfere with normal breathing. It can affect just upper respiratory system, which starts at sinuses and ends at your vocal chords and also affect just lower respiratory system. ${ }^{1}$ Acute respiratory infection prevents the body from getting oxygen and can result in death. Person suffering from this condition needs medical assistance immediately. Also, these infections are infectious, which means they can spread from one person to another. The disease is quite widespread. It is particularly dangerous for children, older adults, and people with immune system disorders. According to the World Health Organization (WHO), acute respiratory infections kill an estimated 2.6 million children annually every year worldwide. The early symptoms of acute respiratory infection usually appear in the nose and upper lungs and If the disease advances, there may be high fever and chills. Causes of ARI can be Pneumococcus, Adenoviruses and Rhinoviruses mainly., ${ }^{2,3}$

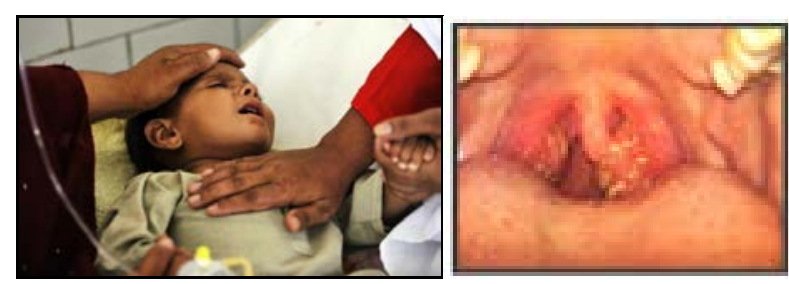

Figure 1a and 1b: ARI in infants

WHO Classified ARI of under 5 year children for early diagnosis and proper management. ARI of under 5 year children are classified into 1 . No pneumonia, 2. Pneumonia, 3. Severe pneumonia and 4. Very severe pneumonia. Breast feeding is an important determinant of child health in the prevention of malnutrition and infection. In many cultures the introduction of other foods especially cereals occurred months or even years before the cessation of breast feeding. Early weaning may be defined as introduction of other foods before the age of 5 months. It is clear that the introduction of weaning foods before 5 months of age is inappropriate and the predominant age of supplementary feeds is associated with malnutrition of infants. Infants are the children of less than 1 year of age. Early weaning may cause malnutrition to infant and makes them susceptible to infection most commonly ARI. In Bangladesh, Infectious diseases such as diarrhoea and acute respiratory infections are the main cause of mortality and morbidity in infants aged less than one year. The importance of breast feeding in the prevention of infectious diseases during infancy is well known. In this study our main goal is to evaluate the causative relationship between ARI of infant and weaning status of infants admitted in selected Hospitals of Rajshahi.

\section{Objective}

\section{General Objective:}

$>$ To assess the association between acute respiratory tract infection (ARI) of infants and weaning status of infants admitted in selected Hospitals of Rajshahi.

\section{Specific Objective:}

$>$ To assess the proportion of early weaning among the infants having ARI

$>$ To evaluate the proportion of early weaning among the infants without having ARI.

$>$ To find out socio-economic status of the respondents.

$>$ To identify the association between ARI and early weaning practice.

\section{Methodology:}

Study Type:

$>$ It was a case control study.

\section{Study Period and Place:}

$>$ This study was conducted in Department of Community Medicine, Rajshahi Medical College from date of 01/02/13 to 02/02/14.

\section{Sample Size:}

$>$ In this study total 230 patients was taken among them case 100 and control 130.

\section{Sample technique:}


During Study sampling was carried out from the in-patient department of both Rajshahi Medical College Hospital and Rajshahi Shishu Hospital accordingly maintaining the register book of admission and by using simple random sampling technique applying among the ARI infected infants of the whole admitted patient. Babies of control group were selected from the surrounding areas.

\section{Data collection procedure:}

$>$ First of all the permission from the hospital authority was obtained to carry out the research work. Then the study, its objectives, methodology and benefit and risk to the patient were explained to the mother of the infants. After taking informed consent from the mother, data were collected from the mothers according to objectives of the study by a partially structured questionnaire. Data were collected by the researcher herself from the mother by face to face interview. They were allowed to answer the question by their own judgment.

\section{Data analysis procedure:}

First data were edited to the validity and consistency of the data. After proper verification data were coded and entered into computer by using SPSS software programmes. Descriptive analysis was done by percentage, mean and standard deviation. Association was observed by appropriate statistical test at 95\% confidence interval eg. odds ratio, Chi square, t-test .

\section{Result}

During the study a total number of 230 respondents were interviewed according to a pretested questionnaire among which 100 respondents were taken as cases and 130 as controls.

In table- 1 shows distribution of cases and controls by baby's demographic characteristics where the case group $61.0 \%$ babies were above 6 months of age and $39.0 \%$ babies were less than 6 months of age. Within the control group $77.7 \%$ babies were more than 6 months of age and $22.3 \%$ babies were less than 6 months of age. The mean age of the babies was $1.70 \pm 0.45$ months. The following table is given below:

Table- 1: Age of the babies (in months)

\begin{tabular}{|c|c|c|c|}
\hline \multirow[t]{3}{*}{ Samples } & \multicolumn{2}{|c|}{ Age of the respondents } & Total \\
\hline & $<6$ months & $>6$ months & \\
\hline & No (\%) & No (\%) & \\
\hline Case & $39(39 \%)$ & $61(61 \%)$ & 100 (43.5\%) \\
\hline Control & $29(22.3 \%)$ & 101(77.7\%) & $130(56.5 \%)$ \\
\hline Total & $68(29.6 \%)$ & $162(70.4 \%)$ & $230(100 \%)$ \\
\hline
\end{tabular}

In figure- 2 shows $66 \%$ babies were female and $34 \%$ babies were male. Majority of the babies within the case

Were female and for control group $63 \%$ babies were female and $37 \%$ babies were male. Majority of the babies within the control group were female. The following figure is given below: 


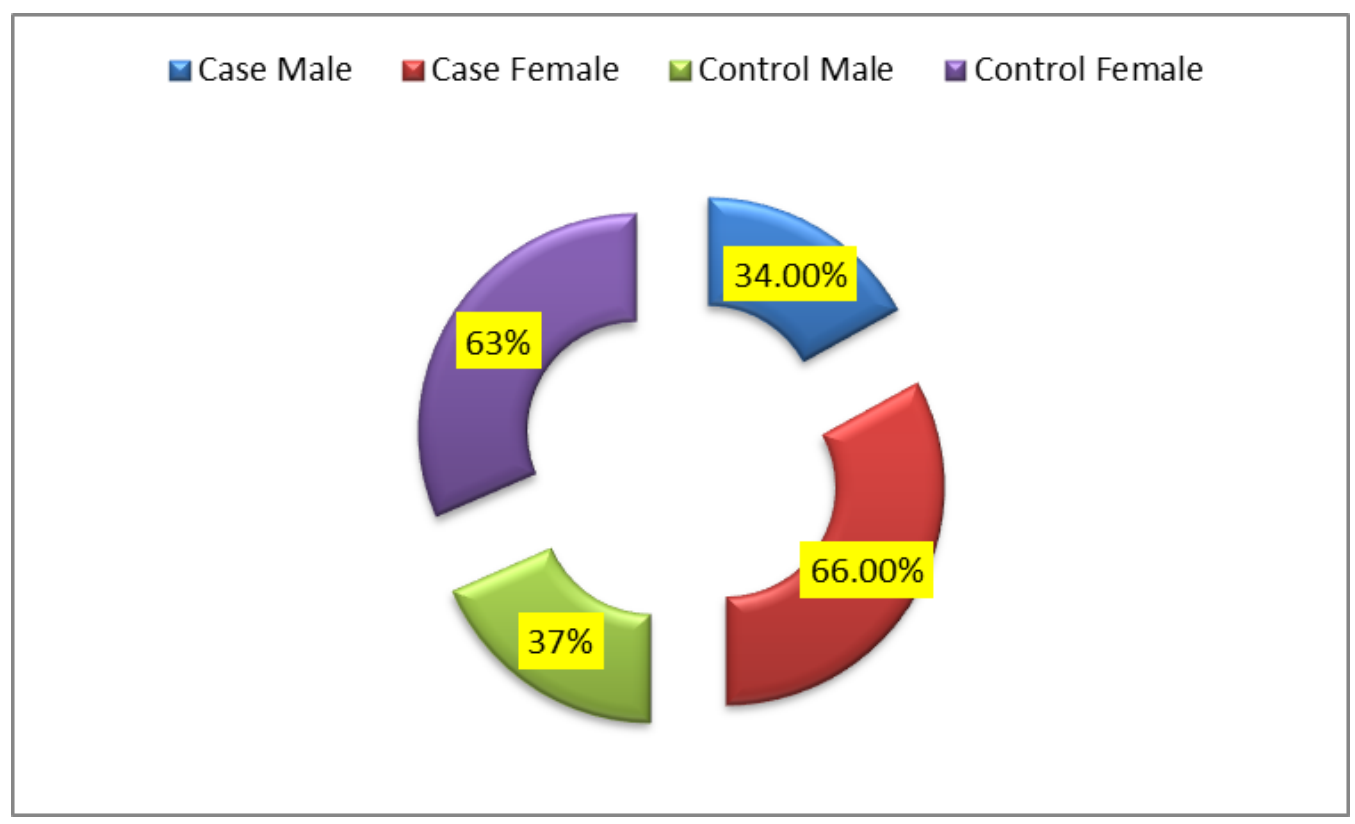

Figure -2: Sex of the babies

In table-2 shows Weaning status of the infants of both case and control group where the case group 68.0\% babies were weaned earlier 5 months, 20.0\% babies were weaned at 6 months and $12.0 \%$ babies were weaned more than 7 months. Within the control group 26.9\% babies were weaned at earlier 5 months, $59.2 \%$ babies were weaned at 6 months and 13.8\% babies were weaned more than 7 months. The association between acute respiratory infections (ARI and weaning status of the infants was statistically significant $(\mathrm{P}<0.001)$.The following table is given below:

Table-2: Weaning status of the infants of both case and control group.

\begin{tabular}{|c|c|c|c|c|}
\hline \multirow[t]{2}{*}{ Sample } & \multicolumn{3}{|c|}{ Weaning status of the infants } & \multirow[t]{2}{*}{ Total } \\
\hline & $\begin{array}{c}\begin{array}{c}\text { Earlier }<5 \\
\text { months }\end{array} \\
\text { No (\%) }\end{array}$ & $\begin{array}{c}\text { at } 6 \text { months } \\
\mathrm{N}(\%)\end{array}$ & $\begin{array}{c}>7 \text { months } \\
\text { No (\%) }\end{array}$ & \\
\hline Case & $68(68.0 \%)$ & $20(20.0 \%)$ & $12(12 \%)$ & 100 (43.5\%) \\
\hline Control & 35 (26.9\%) & 77 (59.2\%) & $18(13.8 \%)$ & 130 (56.5\%) \\
\hline Total & $103(44.8 \%)$ & $97(42.2 \%)$ & $30(13.0 \%)$ & $230(100 \%)$ \\
\hline
\end{tabular}

In figure-3 shows Religion of the mothers of infants where In Case group 80\% mothers were Muslim and 20\% mothers were Hindus and 82\% mothers were Muslim and 18\% mothers were Hindus for Control group. The follow figure is give below: 


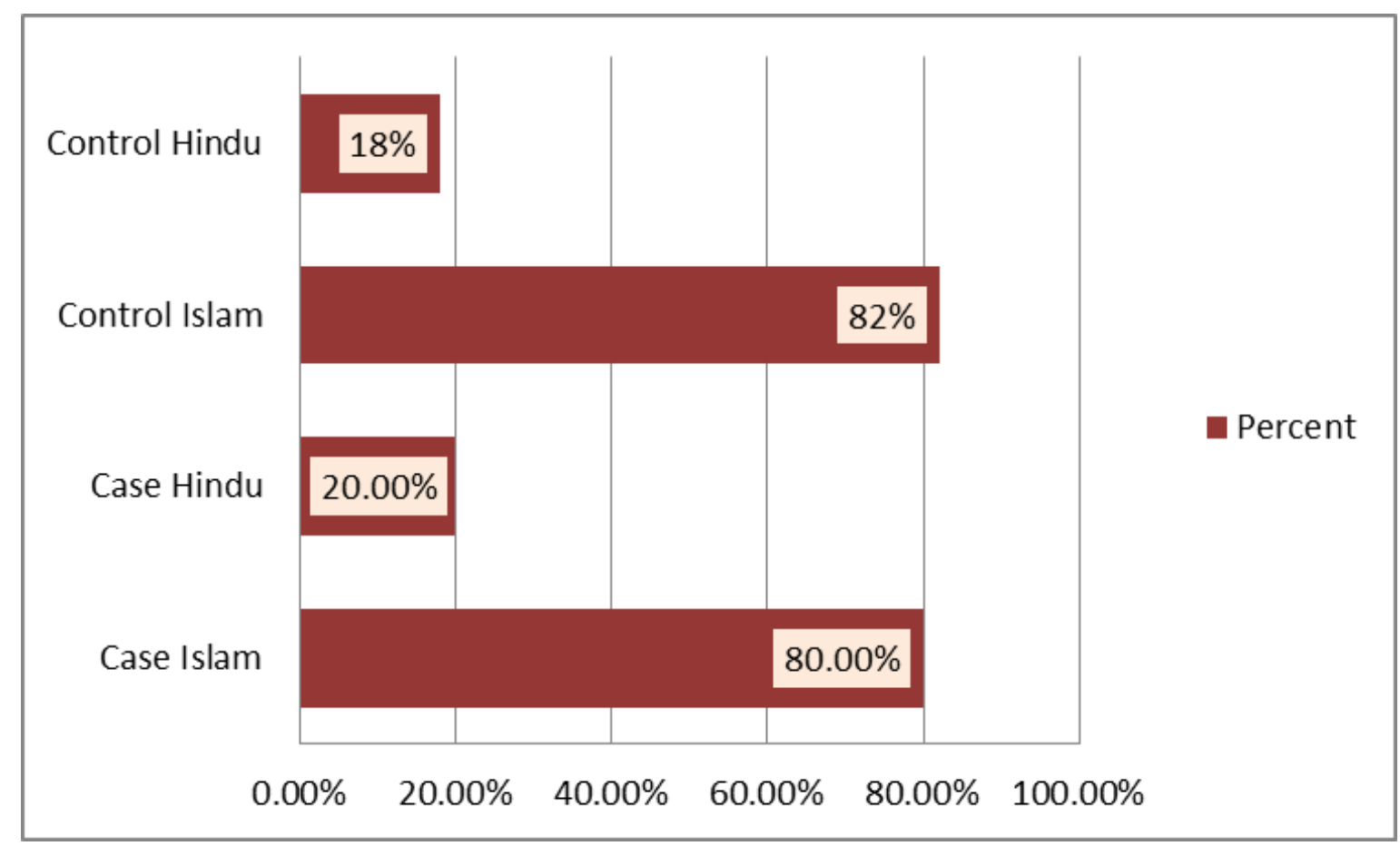

Figure -3: Religion of the infant mothers.

In table-3 shows Educational level of mothers of both case and control group where the case group 18.7\% mothers were illiterate, $6.1 \%$ mothers were having educational qualification of class I-V, $16.1 \%$ mothers were having education level of class XI-XII and 2.6\% mother were having graduation and above degree. Within the control group $25.7 \%$ mothers were illiterate, $14.8 \%$ mothers were having educational level of class I-V, $12.6 \%$ mothers were having class VI-XII degree and $3.5 \%$ were having graduation and above. The following table is given below:

Table-3: Educational level of mothers of both case and control group

\begin{tabular}{|c|c|c|c|c|c|}
\hline \multirow[t]{2}{*}{ Sample } & \multicolumn{4}{|c|}{ Education status of mothers } & \multirow[t]{2}{*}{ Total } \\
\hline & Illiterate & Class I-V & Class VI-XII & $\begin{array}{c}\text { Graduate and } \\
\text { above }\end{array}$ & \\
\hline Case & $43(18.7 \%)$ & $14(6.1 \%)$ & $37(16.1 \%)$ & $6(2.6 \%)$ & $100(44.5 \%)$ \\
\hline Control & $59(25.7 \%$ & $34(14.8 \%)$ & $29(12.6 \%)$ & $8(3.5 \%)$ & $130(56.5 \%)$ \\
\hline Total & $102(44.3 \%)$ & $48(20.9 \%)$ & $66(28.7 \%)$ & $14(6.1 \%)$ & $230(100 \%)$ \\
\hline
\end{tabular}

In figure-4 shows type of a family where for both Case and control group nuclear family number is greater than joint family. The following figure is given below: 


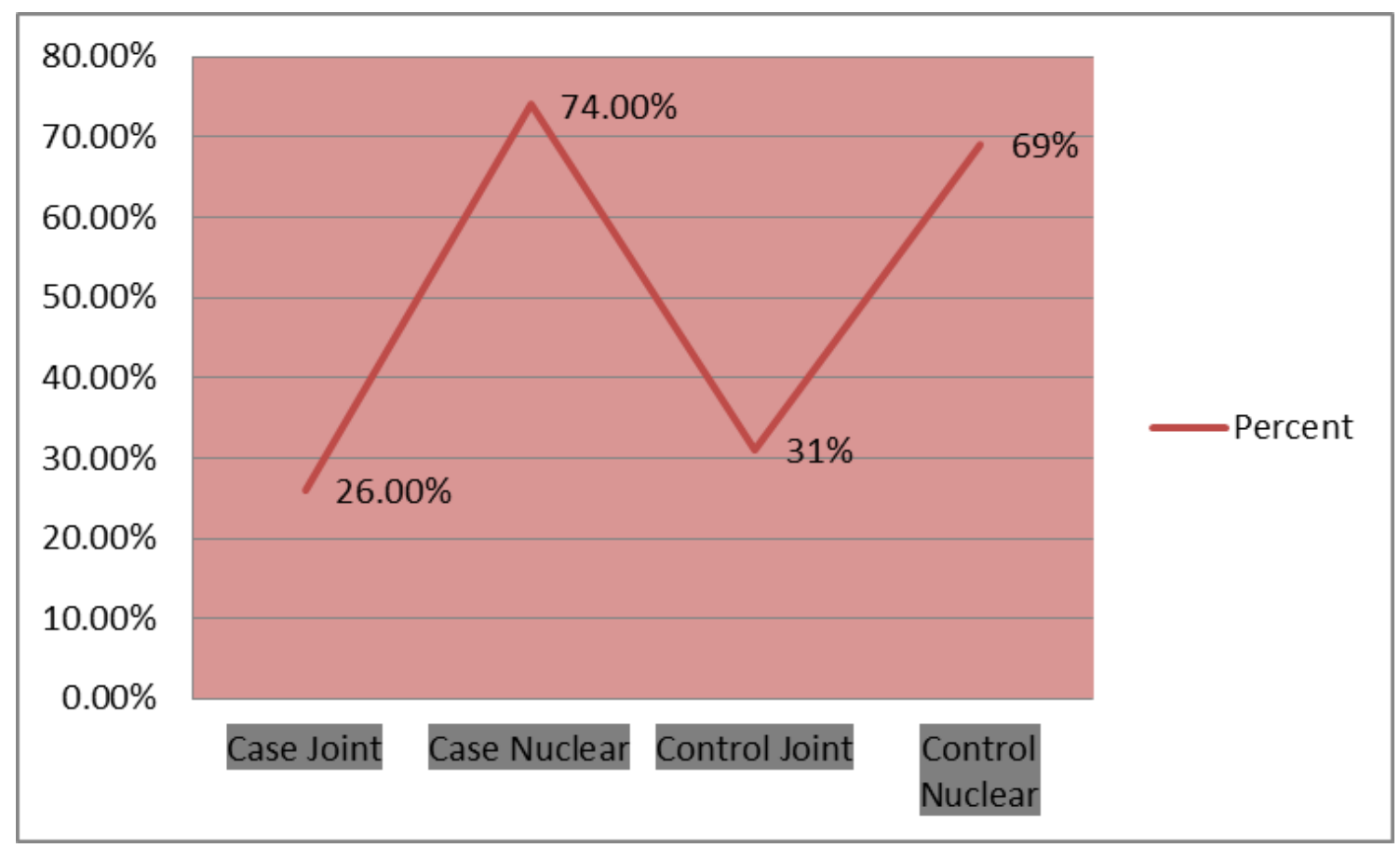

Figure-4: Types of a family

Table-5 shows Occupation of the respondents (mothers or care givers) where within the case group majority of the mothers were housewives, 3\% mothers were day laborer, 3.9\% were having other occupation and there was no service holder. Within the control group majority of the mothers were housewives, 5.7\% mothers were day laborer, $1.7 \%$ mothers were service holder, and $3.5 \%$ mothers were having other occupations. The following table is given below:

Table-5; Occupation of the respondents (mothers or care givers)

\begin{tabular}{lccccc} 
Sample & \multicolumn{2}{c}{ Occupation of the mothers } & Total \\
& Service holder & Day Labourer & Housewives & Others & \\
Case & $00(0 \%)$ & $7(3.0 \%)$ & $84(36.5 \%)$ & $9(3.9 \%)$ & $100(43.5 \%)$ \\
Control & $4(1.7 \%)$ & $13(5.7 \%)$ & $105(45.7 \%)$ & $8(3.5 \%)$ & $130(56.6 \%)$ \\
Total & $4(1.7 \%)$ & $20(8.7 \%)$ & $189(82.2 \%)$ & $17(7.4 \%)$ & $230(100 \%)$
\end{tabular}

In figure- 5 shows Place of residence of both case and control group where case group $49.0 \%$ families were living in urban area and 51.0\% families were living in rural area. Within the control group, 66.2\% families were living in urban area and $33.8 \%$ families were living in rural area. The following figure is given below: 


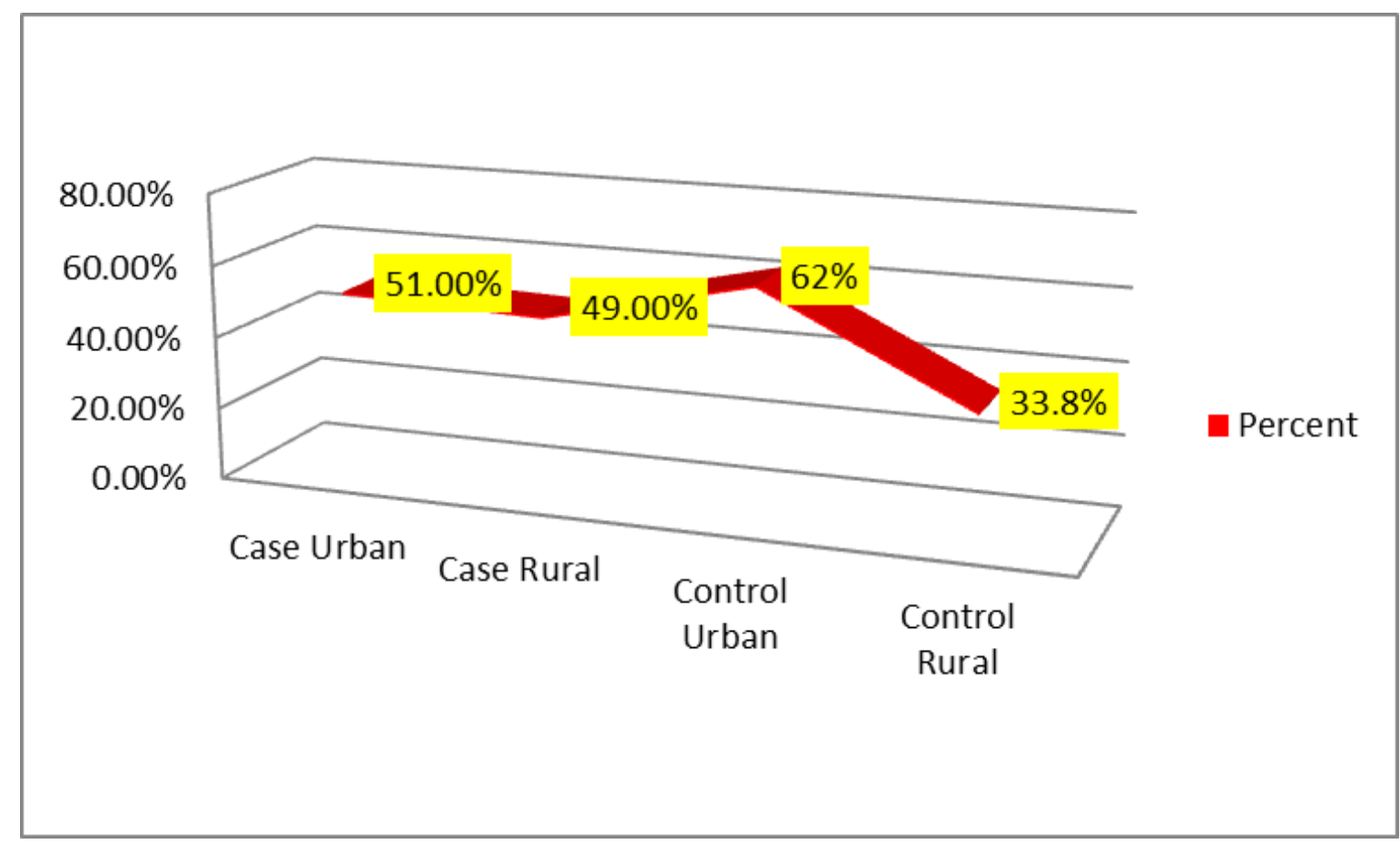

Figure-5: Place of residence of both case and control group

In table- 6 shows Monthly family income of the respondents of both case and control group where the case group $44 \%$ families were having monthly family income of less than 5000 Taka, 50\% families were having 500-10,000 Taka and 6\% families were having more than 10,000 Taka of monthly family income. With the control group 31.5\% families were having monthly family income of less than 5000 Taka, 58.5\% families were having 5000-10,000 Taka and 10\% families were having more than 10,000 Taka. The Following table is given below;

Table-6: Monthly family income of the respondents of both case and control group.

\begin{tabular}{|c|cccc} 
Sample & \multicolumn{3}{|c}{ Monthly family income } & Total \\
& $<5000$ Taka & $5000-10,000$ Taka & $>10,000$ Taka & \\
\hline Case & $44(44.0 \%)$ & $50(50.0 \%)$ & $6(6.0 \%)$ & $\mathbf{1 0 0}(\mathbf{4 3 . 5 \% )}$ \\
\hline Control & $41(31.5 \%)$ & $76(58.5 \%)$ & $13(10.6 \%)$ & $\mathbf{1 3 0 ( 5 6 . 5 \% )}$ \\
\hline Total & $\mathbf{8 5 ( 3 7 . 0 \% )}$ & $\mathbf{1 2 6}(54.8 \%)$ & $\mathbf{1 9 ( 8 . 3 \% )}$ & $\mathbf{2 3 0 ( 1 0 0 \% )}$ \\
\hline
\end{tabular}

In figure -6 shows Immunization status of the babies of both case and control group where for case group $88.0 \%$ babies were complete immunized and $12.0 \%$ babies were incompletely immunized. Where as in Control group $100.0 \%$ babies were completely immunized and $0.0 \%$ babies were incompletely immunized. 


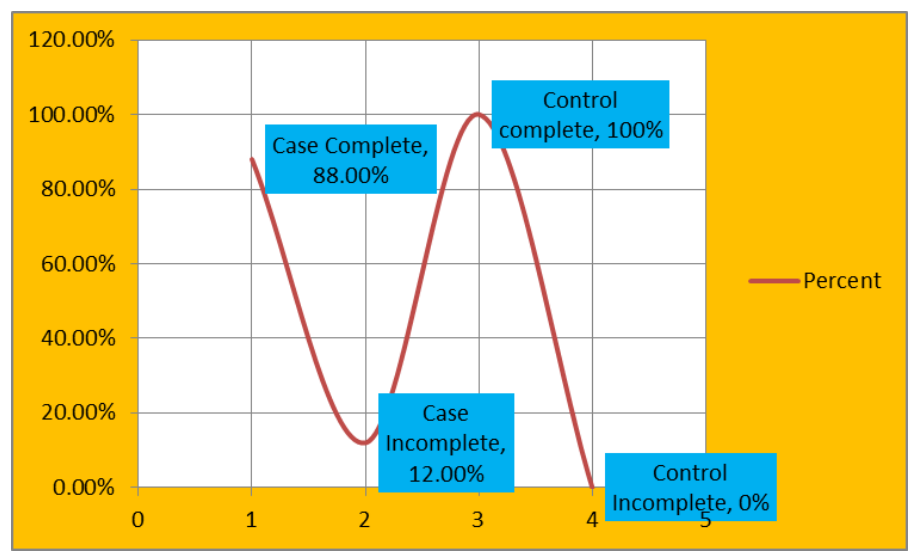

Figure-6: Immunization status of the babies of both case and control group

In table-7 shows Type of weaning food of the infants of both case and control group where case group $44.7 \%$ babies who were weaned with Cow's milk developed ARI. Within the control group 71.3\% babies were weaned with mashed rice, the association between occurrence of ARI and type of weaning food was statistically significant $((\mathrm{P}<0.001)$. The following table is given below:

Table-7: Type of weaning food of the infants of both case and control group

\begin{tabular}{|c|c|c|c|c|c|c|c|}
\hline \multirow[t]{2}{*}{ Sample } & \multicolumn{6}{|c|}{ Type of weaning food } & \multirow[t]{2}{*}{ Total } \\
\hline & $\begin{array}{c}\text { Mashed } \\
\text { Rice }\end{array}$ & Multiple & $\begin{array}{c}\text { Half } \\
\text { boiled egg }\end{array}$ & $\begin{array}{l}\text { Cow's } \\
\text { milk }\end{array}$ & Others & $\begin{array}{c}\text { Not } \\
\text { applicable }\end{array}$ & \\
\hline Case & $23(10.0 \%)$ & $12(5.2 \%)$ & $0(00 \%)$ & $38(16.5 \%)$ & $15(6.5 \%)$ & $12(5.2 \%)$ & $100(43.5 \%)$ \\
\hline Control & $57(24.8 \%)$ & $5(2.2 \%)$ & $8(3.5 \%)$ & $47(20.4 \%)$ & $8(3.5 \%)$ & $5(2.2 \%)$ & $130(56.5 \%)$ \\
\hline Total & $80(34.8 \%)$ & $17(7.4 \%)$ & $8(3.5 \%)$ & $85(27.0 \%)$ & $23(10.0 \%)$ & $17(7.4 \%)$ & $230(100 \%)$ \\
\hline
\end{tabular}

In table-8 shows EBF practiced properly or not of both case and control group where case group, 32.0\% babies were practiced EBF properly and within the control group $73.1 \%$ babies were practiced EBF properly. Majority of the babies were practiced EBF properly within the control group.

Table-8: EBF practiced properly or not of both case and control group

\begin{tabular}{cccc} 
Sample & \multicolumn{2}{c}{ EBF practiced properly } & Total \\
& Yes & No & \\
Case & $32(32.0 \%) \%)$ & $68(68.0 \%) \%)$ & $100(43.5 \%)$ \\
Control & $95(73.1 \%)$ & $35(26.9 \%)$ & $130(56.5 \%)$ \\
Total & $127(55.2 \%)$ & $103(44.8 \%)$ & $230(100 \%)$
\end{tabular}


Figure-8 shows Association between place of Residence and occurrence of ARI of both case and control where $51.0 \%$ families those were living in rural area developed ARI and $66.2 \%$ families those were living in urban area having no ARI. The association between occurrence of ARI and place of residence was statistically significant $(\mathrm{P}<0.005)$. $\mathrm{F}$ The following figure is given below:

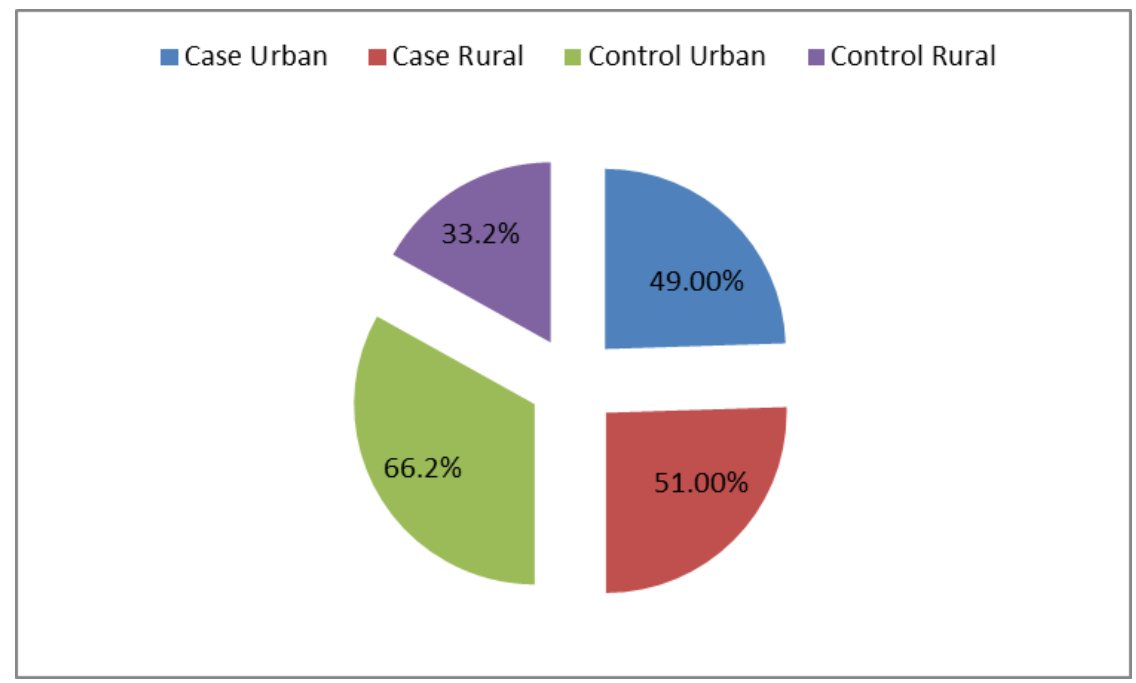

Figure-8: Association between place of Residence and occurrence of ARI of both case and control.

In table-9 shows Relationship between proper exclusive breast feeding (EBF) and occurrence of ARI of both case and control group where the case group majority of the babies (68.0\%) those who were not EBF practiced properly developed ARI within the control group majority of the babies $(73.1 \%)$ were EBF practiced properly having no ARI. The association between occurrence of ARI and EBF practiced properly was statistically significant $(\mathrm{P}<0.001)$. The following table is given below:

Table-9: Relationship between proper exclusive breast feeding (EBF) and occurrence of ARI of both case and control Group

\begin{tabular}{cccc} 
Sample & $\begin{array}{c}\text { EBF practiced } \\
\text { properly }\end{array}$ & Total & Sample \\
\hline Case & Yes & No & Case \\
Control & $32(32.0 \%)$ & $68(68.0 \%)$ & Control \\
Total & $95(73.1 \%)$ & $35(26.9 \%)$ & Total \\
\hline
\end{tabular}

In figure-10 shows Relationship between immunization status of the infants and occurrence of ARI of both case and control group where case group $12.0 \%$ babies those who were not completely immunized developed ARI. Within the control group $100.0 \%$ babies were complete immunized and they did not developed ARI. The association between immunization status of the infants and occurrence of ARI was statistically significant $(\mathrm{P}<0.001)$.The following figure is given below: 


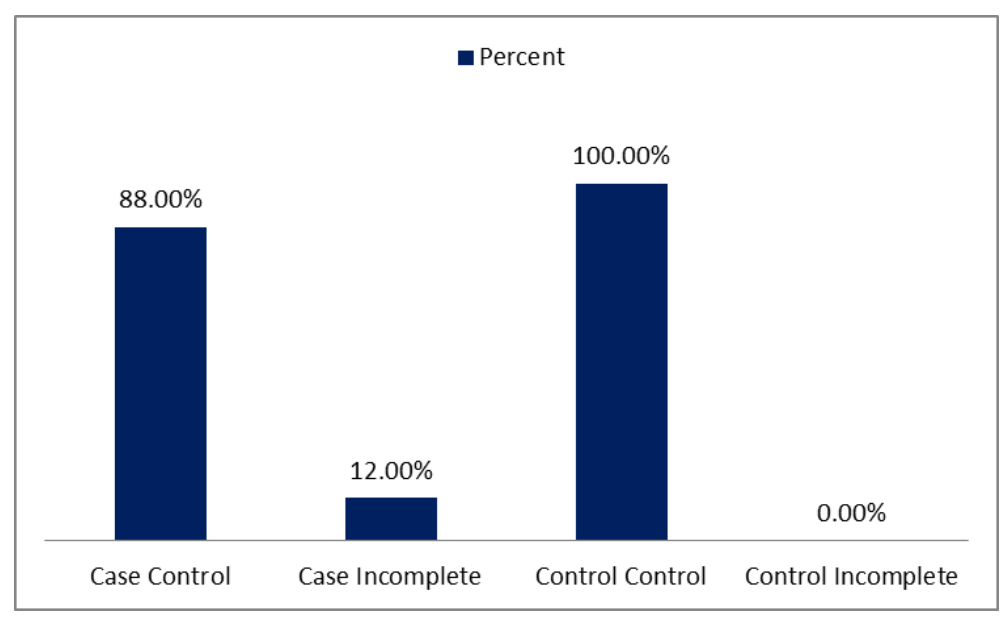

Figure-10: Relationship between immunization status of the infants and occurrence of ARI of both case and control group

Table-11 shows Association between early weaning and standard weaning status of infants of both case and control group where $68 \%$ of babies who were weaned earlier developed ARI and $26.9 \%$ of babies who were weaned earlier having no ARI. The odd ratio was=7.5. The occurrence of ARI among cases in this 7.5 times more than that among the controls. The following table is given below:

Table-11: Association between early weaning and standard weaning status of infants of both case and control group.

\begin{tabular}{ccc} 
Sample & \multicolumn{2}{c}{ Time of weaning by mothers } \\
& Earlier $(<5$ months $)$ & At 6 months \\
Case & $68(68 \%)$ & $20(20 \%)$ \\
Control & $35(26.9 \%)$ & $77(59.2 \%)$
\end{tabular}

Table-12 shows Association between late weaning and standard weaning status of infants of both case and control group where odd ratio between late weaning and standard weaning was 2.5. So, the babies who were weaned late 7 months about 2.5 times more prone to developed ARI. The following table is given below:

Table-12: Association between late weaning and standard weaning status of infants of both case and control group

\begin{tabular}{ccc} 
Sample & \multicolumn{2}{c}{ Late weaning and standard weaning status } \\
& Late weaning $>7$ months & Standard weaning \\
Case & $12(12.0 \%)$ & $20(20.0 \%)$ \\
Control & $18(13.8 \%)$ & $77(59.2 \%)$
\end{tabular}




\section{Discussion}

The study showed that within the case group majority of the babies $68 \%$ were weaned earlier ( $<5$ months) of age and within the control group majority of $26.9 \%$ of the infants were weaned at 6 months of age. The association between weaning status of infants and occurrence of ARI among them was statistically significant $(\mathrm{P}<0.001)$.Many studies reported that a high prevalence of ARI among under-five children and pointed out various socio-demographic, nutritional and environmental factors as the modifiable risk factors. ${ }^{5}$ The overall prevalence of ARI was found to be $26.22 \%$. Also in other studies conducted a study to find out association between feeding practice and pneumonia in under 5 children. Regarding mode of feeding in the present study breast feeding, period of exclusive breast feeding, colostrum intake, bottle feeding practice and weaning practice was assessed There was statistically significant difference $(\mathrm{P}=0.002)$ between breast fed and non-breast fed children in developing severe pneumonia. Non-breast fed were found to be more prone to developed severe pneumonia than those who were breast fed (54.4\%). Regarding educational status of the mother it was found that within the case group $18.7 \%$ women were illiterate and within the control group $25.7 \%$ women were illiterate. So, majority of the mothers were illiterate. The current literacy rate in Bangladesh is about $46.0 \%$. It was found that within the case group majority of the mothers were housewives and also in control group majority of the mothers were housewives and within the case group majority of families were nuclear. A study conducted by many scientists showed that the prevalence of ARI was found to be $22 \%$, it was higher in low social class (III, IV and V), illiterate mothers $(24.4 \%)$ and mothers having education primary (23.9\%). ${ }^{7}$ Prevalence of ARI was lower in urban area (17.2\%) as compare to rural area (26.8\%) (combined is 22\%). In this study we found that occurrence of ARI was 22.2\% among the infants of the families who were living in rural area. Regarding type of weaning food of infants it was found that within the case group majority of the babies were weaned by Cow's milk. In this study it was food there was a high tendency to use Cow's milk as weaning food. So, we should discourage mother not to give their babies' Cow's milk alone as weaning food. It was found that babies of the case group were incompletely immunized and within the control group majority of the babies were completely immunized. Regarding type of weaning food of infants it was found that within the case group majority of the babies were weaned. Regarding the relationship of exclusive breast feeding practice and occurrence of ARI among infants it was found that majority of the babies who were not EBF practiced properly developed ARI. The association between proper exclusive breast feeding and occurrence of ARI was statistically significant $(\mathrm{P}<0.001)$. Many studies conducted that exclusive or predominant breastfeeding case rates of morbidity significantly low in this region of rural Bangladesh because breastfeeding is almost universal in Bangladesh; the rates of exclusive breastfeeding remain low.

\section{Recommendations}

The following recommendations might be made on the basis of the study outcomes:

$>$ Babies should not be weaned earlier less than 5 months because of there is a higher risk to developed ARI.

> Babies should be exclusively breast fed upto 6 months of age than gradually weaning should be done by introducing complementary foods such as mashed rice. Cow's milk as weaning food should be avoided.

\section{Conclusion}

After many studies and analysis we found that within the case group majority of the babies who were not exclusively breast fed developed ARI and the association between occurrence of ARI and EBF was statistically significant $(\mathrm{P}<0.001)$. So, from the information we came to a conclusion that proportion of ARI is more among the infants who were weaned earlier 


\section{References}

1. Pavel, Omer Fayshal. "Acute Respiratory Infection (ARI)." (2016). Bangladesh Today.

2. Williams, Brian G., Eleanor Gouws, Cynthia BoschiPinto, Jennifer Bryce, and Christopher Dye. "Estimates of world-wide distribution of child deaths from acute respiratory infections." The Lancet infectious diseases 2, no. 1 (2002): 25-32.

3. Leowski, Jerzy. "Mortality from acute respiratory infections in children under 5 years of age: global estimates." (1986). PAHO epidemiological Bulletin

4. Fazana I. Ratna S. Arup D, Sumit N and Ranabir P (2013), 'Profiling Acute Respiratory Tract infections in children from Assam, India'. Journal of global Infectious Diseases 2013; 5(1), 8-14

5. Daniel W. Selc (2007), 'Evolution of Infant and Young Child Feeding: Implications for Contemporary Public Health', Annu. Rev. Nutr: Vol.27, Pp 123-48.

6. Bipin Prajapati, Nitiben Talsania Sonaliya $\mathrm{K} \mathrm{N}$, (2011), 'A study on prevalence of acute respiratory Tract infections (ARI) in under five children in Urban and rural communities of Ahmadabad District, Gujarat', National Journal of Community Medicine Vol. 2, Issue 2, Pp 255-259. 